UCRL-JC-119579

PREPRINT

\title{
Three Dimensional Simulations of a Small Induction Recirculator Accelerator
}

\author{
D. P. Grote, A. Friedman, and I. Haber, \\ Lawrence Livermore National Laboratory, University of California \\ P.O. Box 5508, L-440, Livermore California 94550, USA
}

'Naval Research Laboratory,

Washington DC 20375, USA

RECEIVED

OCT 131995

This paper was prepared for submittal to the

OSTI

Proceedings of the IEEE 1995 Particle Accelerator Conference

Dallas, Texas

May 1-5, 1995

April 24, 1995

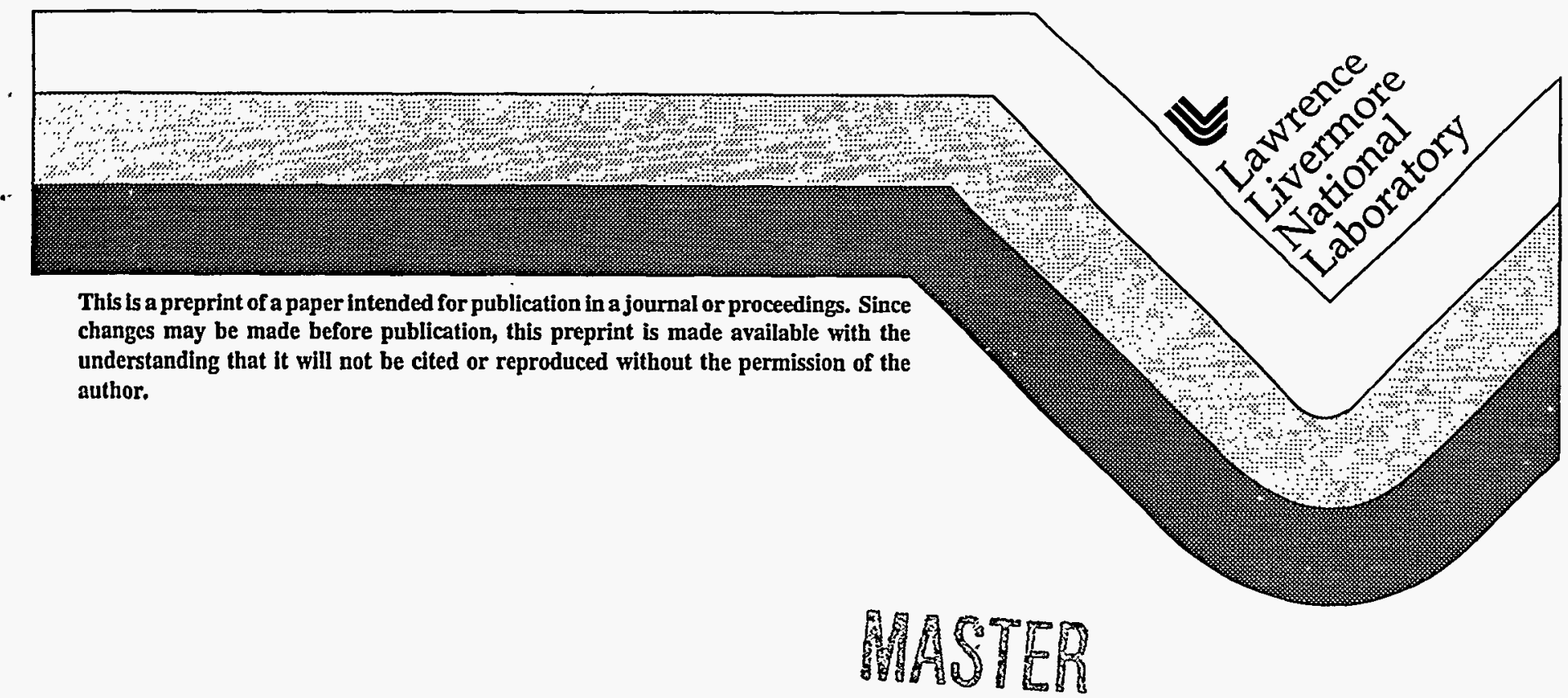




\section{DISCLAIMER}

Portions of this document may be illegible in electronic image products. Images are produced from the best available original document. 


\title{
THREE DIMENSIONAL SIMULATIONS OF A SMALL INDUCTION RECIRCULATOR ACCELERATOR*
}

\author{
D. P. Grote and A. Friedman, Lawrence Livermore National Laboratory, Livermore, CA 94550 USA, \\ and I. Haber, Naval Research Laboratory, Washington, DC 20375-5346 USA
}

\section{Abstract}

A recirculating induction accelerator has potential cost advantages for a heavy-ion fusion driver. In order to explore the physics and technological issues, a small prototype recirculator is being built[1]. The three dimensional particlein-cell code, WARP3d[2], is being used in the design and analysis of the experiments. WARP3d is used to examine the behavior of the beam in the electric dipoles and in the non-linear fields associated with the accelerator lattice elements. The dipoles have focusing and fringe fields which can adversely affect the beam quality. Both single particle and full beam dynamics are examined in the dipoles using realistic geometries. Dipole plate designs which minimize the adverse effects are described. The non-linear fields associated with the permanent magnetic quadrupoles have been included in the simulations. They were found to have little effect on the quality of the beam.

\section{WARP3d}

The realization of heavy ion driven inertial fusion requires a detailed quantitative understanding of the behavior of high-current ion beams. The three-dimensional particle-in-cell simulation code WARP3d is used to study the transport and acceleration of space-charge dominated ion beams in present-day and near-term experiments, and in fusion drivers. WARP3d includes a number of novel techniques and capabilities that both enhance its performance and make it applicable to a wide range of problems. The techniques include a method for sub-grid scale resolution of conducting boundary locations in the solution of Poisson's equation, a warped coordinate system to model accelerator bends, and "residence corrections" to ensure that particles receive the correct impulse from sharp-edged finite-length external fields whose edges are intercepted at different times during a time step. The capabilities include the ability to model the time-dependent accelerator gap fields used for axial beam confinement and compression, application of field multipole components as a function of axial location (or along with the residence correction for hard-edged elements) and multi-species beams. WARP3d is built on top of the Basis[3] system which allows interactive (run time) control of the code and access to its internal database.

\footnotetext{
"Work performed under auspices of U.S. DoE by LLNL and NRL under contracts W-7405-ENG-48 and DE-AI02-93ER40799, DEAI02-94ER54232.
}

\section{Induction Recirculator}

A small scale recirculating induction accelerator is being built to study beam dynamics in a recirculating accelerator and to examine various engineering design issues. The accelerator was designed to have beam dimensionless parameters relevant to those of a driver. With a circumference of $14.4 \mathrm{~m}$, there are 40 half lattice periods, each with a permanent magnetic quadrupole, an electric dipole, and an induction acceleration gap. A beam of singly charged potassium ions will be accelerated from 80 to $320 \mathrm{keV}$ over 15 laps, at a current increasing from .2 to .8 Amps. With a low emittance, the beam behavior is space-charge dominated. As designed, the phase advance is initially depressed from $72^{\circ}$ to $16^{\circ}$.

\section{Electric Dipoles}

To make the construction of the recirculator easier and less costly, electric dipole plates were chosen to bend the beam. The length of the plate is constrained by the amount of space available for focusing and bending the beam. The maximum length that would fit is roughly one and a half times the plate separation. Also, the ends of the plates are near the structure supporting the quadrupole magnets. With the height of the plates limited similarly, the plates are nearly square. As a result of the constraints, there are significant nonlinear fields produced by the dipole plates.

Analysis of the dipoles' effect on the behavior of the beam calls for self-consistent simulations which include both the fields produced by the plates and the self-fields of the beams. WARP3d was used to carry out the simulations. Figure 1 shows the layout of the half lattice periods as used in the simulations.

The dominant nonlinear component of the field from the dipole plates is the sextupole component, $\phi \propto r^{3} \cos (3 \theta)$. With flat plates, the sextupole rapidly alters the beam shape. After one quarter of a lap the transverse profile of the beam becomes markedly triangular. The addition of ridges on the top and bottom of the plates can alter the sextupole component. The ridges that are typically added to dipole plates have a height that is $10 \%$ of the plate separation and minimize the sextupole induced by finite plate height[4]. However, in this case, because the axial fringe fields also introduce a significant sextupole, minimizing the sextupole due to finite plate height only is not satisfactory. The ridges have to be made larger, driving the sign of the sextupole in the center of the plates negative so that the average of the sextupole over the lattice period is minimized. The height of the resultant ridges is roughly $30 \%$ of the plate separation. 


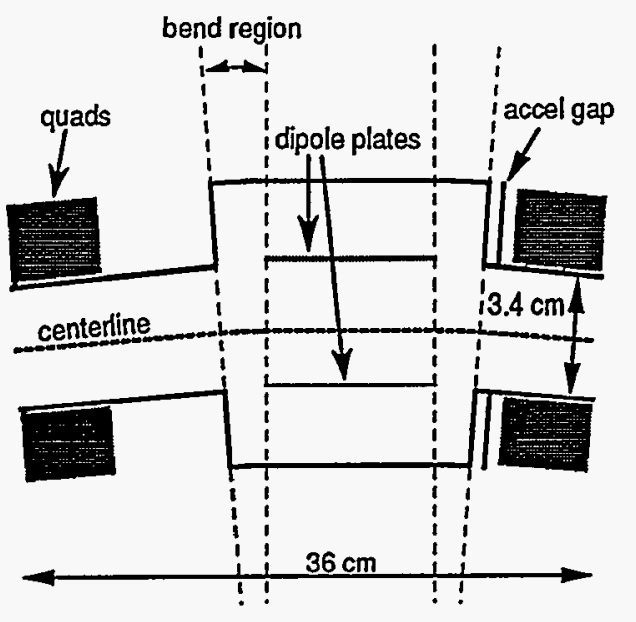

Figure. 1. The top view of the geometry of the recirculator half lattice period as used in WARP3d.

The transverse variation of the potential across the beam in the dipole gives rise to a focusing effect in the plane of the bend. The outer edge of the beam slows down in the dipole and is bent more, whereas the inner edge of the beam speeds up and is bent less. The result is a focusing of the beam. There is also a focusing (or defocusing) in the plane out of the bend from the vertical fringe fields. The combination results in different phase advances in the two planes. Shifting the plates radially, i.e. in the plane of the bend, changes focusing in both transverse directions; an outward shift lowers the in-plane focusing and raises the out-of-plane focusing. The plates can be shifted so that the phase advance is the same in both planes.

The change in potential along the beam centroid in the dipole changes the speed of the beam in the dipole, changing the overall timing of the beam. If the resulting timing change is not included in the timing of the axially confining fields, significant line charge waves will be launched from the ends of the beam. The change in the timing can be controlled by adjusting the radial location of the dipole plates, which changes the location of the potential in the beam relative to ground. There is a radial plate position which minimizes the timing change, avoiding the necessity of having to include the change in the accelerator timing.

Unfortunately, the plate location giving matched phase advances and the plate location giving zero timing change do not coincide. Further plate shaping is therefore needed. With the plates located to minimize the timing change, the in-plane focusing is larger than the out-of-plane focusing. The plates can be curved out of the plane of the bend to increase the vertical focusing.

The plate shape has been tuned to minimize the effects described above. The gridded representation of the plate developed is shown in figure 2. The undepressed phase advances in both planes are near $79^{\circ}$ with a phase advance with quadrupoles only of $72^{\circ}$. With a beam energy of 80 $\mathrm{kV}$, the outer plate is held at $6889 \mathrm{~V}$ and the inner plate is held at $-6262 \mathrm{~V}$. The design was arrived at by iteratively

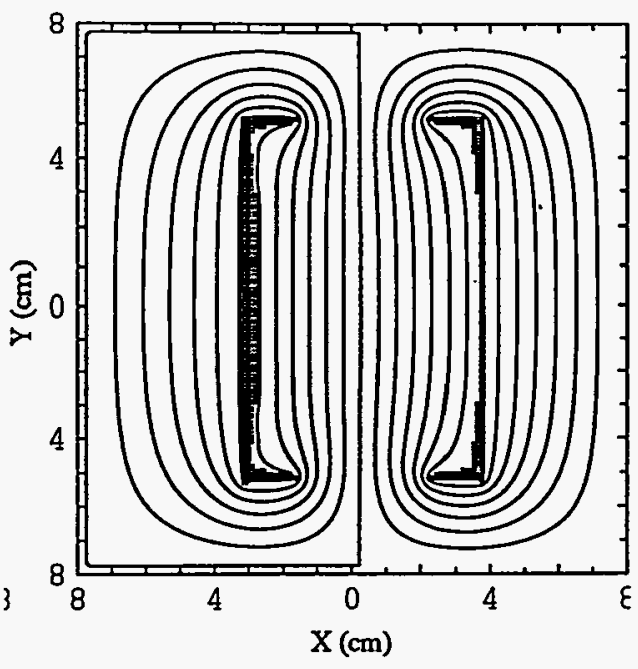

Figure. 2. The optimized design of the dipole plate, minimizing emittance growth. The beam goes into the page and will be bent to the left. The lines are contours of constant potential.

adjusting the curvature and ridges until the in-plane and out-of-plane focusing matched, and then adjusting the radial location of the plates and the voltages on the plate to bring the timing change to zero.

\section{Simulation Results}

WARP3d is being used to simulate the beam in the recirculator for 15 laps. Typically, the beam, which is about 3.5 half-lattice periods or $2 \mu s$ long, is made up of 100,000 simulation particles. The field grid has dimensions $32 \times 16$ in the transverse plane and 128 cells axially, covering four half lattice periods. The symmetry is taken advantage of and only the top half of the accelerator is simulated, allowing the use of half the number of grid cells in the vertical direction. The quadrupoles are modelled as hard edged, with no nonlinear components.

The dipole plates are modelled fully self-consistently except for image fields effects. The field from the dipole plates is calculated with Laplace's equation including the detailed shape of the plates and the surrounding conductors with SOR. During the simulation, the pre-calculated field of the dipole is overlaid on top of the beam self-fields. The selffields are calculated with a 3-D FFT, which is faster than SOR, in a rectangular box with the images on the sides. The transverse dimensions of the box are the same as the plate separation. This only approximates the true images on the dipole plates and quadrupole pipe, but the images are small and the difference is negligible.

The accelerating schedule and axially confining fields give a beam with constant physical length. The accelerating field is ramped up linearly in time when the beam is in the first lap. After that, the field is held constant. The axially confining fields, which are not varied throughout the simulation, are continuously applied to the beam ends.

In the simulations, there is an initial increase in emittance on the order of $50 \%$ as the beam matches itself from a 
a)

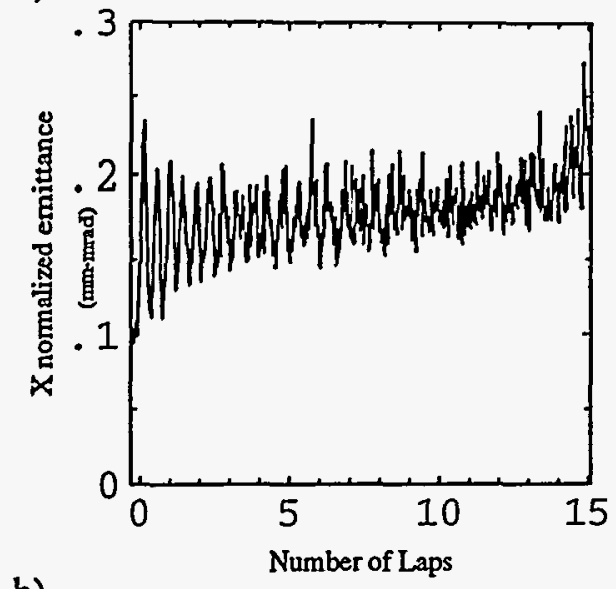

b)

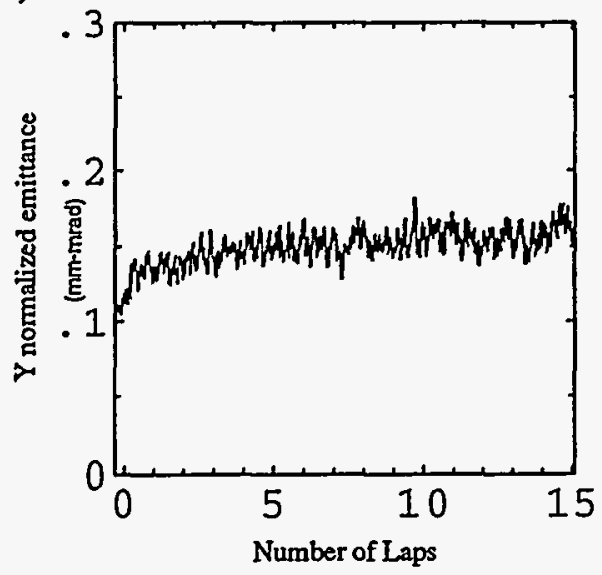

Figure. 3. Time histories of the mid-pulse normalized emittance a) in the plane of the bend and b) out of the plane of the bend with the optimized design of the dipole plates. There is little growth after the initial increase which is due to the beam matching to the bends.

straight path to a curved path. This growth is due to a separation of the particles with differing axial velocities in the bends producing nonlinearities in the space charge[1]. The nonlinearities thermalize producing an increase in emittance. The amount of growth agrees with theoretical analysis[5].

The simulations showed that the behavior of the beam is sensitive to the design of the dipole plates. Flat plates with no ridges very rapidly produce a triangular shaped beam. Shifting the plates by $2 \mathrm{~mm}$ radially without including the resulting timing change in the timing of the axially confining fields produces line charge waves on the order of $10 \%$. When the in-plane and out-of-plane focusing are not matched, the beam becomes elliptical and the emittance grows more rapidly in the direction with the larger size.

Use of the tuned plate shape described in the previous section corrects the problems. In a simulation over the 15 laps, there was little growth in the emittance after the initial growth from matching to the bends. Figure 3 shows the time history of the $x$ and $y$ normalized emittances at the beam mid-pulse. The line charge waves were negligible.
In the experiment, pulse compression will be done to increase the line charge, and the transverse size of the beam will correspondingly grow. The simulations do not yet include pulse compression. Two cases were therefore simulated, one with the size of the simulated beam set to the size of the beam as it enters the recirculator, and the other with it set to the larger size of the beam after it exits the recirculator and after pulse compression. Figure 3 shows the results of the former case. The latter case has the same result, little emittance growth after the initial increase.

\section{Nonlinear Fields of the Quadrupole Magnets}

Estimates were made of the nonlinear fields associated with the permanent magnetic quadrupole design[6]. These fields were included in multi-lap simulations and did not increase the growth of the emittance. These simulations did not include the nonlinear fields which would result from misalignments in the magnet.

\section{Conclusions}

A simulated beam was successfully accelerated in a model of the planned small induction recirculator for 15 laps with minimal growth in transverse normalized emittance. There was little further emittance increase after the initial unavoidable increase due to matching to the bends. Critical to the success was the design of the electric dipole plates. They were designed to minimize the effects resulting from the plates' separation being comparable to their length and from the plates being close to the surrounding conductors. The design also has the in-plane and out-ofplane focusing from the dipoles the same.

\section{References}

[1] A. Friedman et. al., these Proceedings.

[2] A. Friedman, D. P. Grote, and I. Haber, "Threedimensional particle simulation of heavy-ion fusion beams," Phys. Fluids B 4, 2203 (1992).

[3] P. F. Dubois et. al., "The Basis System," LLNL Document M-225 (1988).

[4] Andris Faltens, Private communication (1994)

[5] J. J. Barnard, H. D. Shay, S. S. Yu, A. Friedman, D. P. Grote, "Emittance Growth in Heavy Ion Recirculators," 1992 Linear Accelerator Conference Proceedings, AECL 10728, p. 229 (1992).

[6] Steve Lund, Private communication (1994) 